\title{
Escala para avaliação de resultados de cirurgia estética do abdome
}

\author{
Clinical score scale for outcomes of aesthetic surgery of the abdomen
}

\author{
Alessandra Grassi Salles ${ }^{1}$ \\ Marcus Castro Ferreira ${ }^{2}$ \\ Adelina FÁtima do \\ Nascimento Remigio ${ }^{3}$ \\ Rolf Gemperli ${ }^{4}$
}

Trabalho realizado no Hospital das Clínicas da Faculdade de Medicina da Universidade de São

Paulo, São Paulo, SP, Brasil.

Artigo submetido pelo SGP (Sistema de Gestão de Publicações) da RBCP

Artigo recebido: $16 / 6 / 2010$ Artigo aceito: 4/7/2010

\begin{abstract}
RESUMO
Introdução: A padronização da avaliação de resultados após cirurgia estética é uma dificuldade em Cirurgia Plástica, por ser baseada em critérios geralmente subjetivos. Objetivo: O objetivo deste artigo é apresentar uma escala de uso clínico simples, de fácil reprodução e que forneça critérios objetivos para a avaliação de resultados estéticos de cirurgias plásticas no abdome. Método: A escala foi desenvolvida pela Disciplina de Cirurgia Plástica da Faculdade de Medicina da Universidade de São Paulo. $O$ avaliador dá uma nota $(0=$ insatisfatório, $1=$ regular, $2=$ bom e/ou cicatriz inexistente) para cada um de cinco parâmetros: volume do abdome, contorno lateral, excesso de pele, aspecto do umbigo e qualidade da cicatriz em parede abdominal. Um quadro orienta a pontuação para cada parâmetro. Discussão: A escala é sensível na identificação de diferentes alterações anatômicas no abdome, pode ser utilizada no pré e pós-operatório para comparação de variadas técnicas cirúrgicas, seja abdominoplastia, lipoaspiração e suas variações, ou mesmo para padronizar resultados a serem apresentados em Congressos Médicos ou publicações. A avaliação pode ser feita por fotografias ou pela própria paciente, nas consultas de pré e pós-operatório, documentando de forma objetiva em prontuário a melhora proporcionada pelo procedimento cirúrgico, ferramenta útil como defesa em processos médico-legais.
\end{abstract}

Descritores: Avaliação de Resultados (Cuidados de Saúde). Lipectomia. Cirurgia Plástica. Abdome/cirurgia.

\begin{abstract}
Introduction: The standardization of evaluation of outcomes after aesthetic surgery is still a challenge in Plastic Surgery, being mostly of the times based on subjective criteria. Objective: The purpose of this article is to present a clinical score scale for evaluation of aesthetic results of plastic surgery in the abdomen that is simple, easily reproducible and provides objective criteria. Methods: The scale was developed in the Division of Plastic Surgery, Faculty of Medicine, University of São Paulo. The evaluator gives a score $(0=$ unsatisfactory, $1=$ fair, 2 = good and / or scar absent) for each of five parameters: volume of the abdomen, lateral contour, excess of skin, aspect of navel and quality of the scar. A table helps to choose the score on each parameter. Discussion: The scale is sensitive in identifying different anatomical abnormalities in the abdomen, may be used to compare pre and postoperative results in various surgical techniques, like abdominoplasty, liposuction and its variations, and may help to standardize results presented in meetings or publications. The evaluation can be done using photos or directly with the patient, both before and after surgery appointments, documenting objectively in medical records the improvement provided by the surgical procedures.
\end{abstract}

Keywords: Outcome Assessment (Health Care). Lipectomy. Surgery, Plastic. Abdomen/ surgery.

1. Doutorado pela Faculdade de Medicina da Universidade de São Paulo; Coordenadora do Grupo de Cosmiatria da Divisão de Cirurgia Plástica do Hospital das Clínicas da Faculdade de Medicina da Universidade de São Paulo (HC-FMUSP), São Paulo, SP, Brasil.

2. Professor Titular da Disciplina de Cirurgia Plástica da Faculdade de Medicina da Universidade de São Paulo; Chefe da Divisão de Cirurgia Plástica e Queimaduras do HC-FMUSP, São Paulo, SP, Brasil.

3. Especialista pela Sociedade Brasileira de Cirurgia Plástica; Médica Pesquisadora da Disciplina de Cirurgia Plástica da Faculdade de Medicina da Universidade de São Paulo, São Paulo, SP, Brasil.

4. Professor Livre-Docente pela Faculdade de Medicina da Universidade de São Paulo; Professor Associado da Faculdade de Medicina da Universidade de São Paulo, São Paulo, SP, Brasil. 


\section{INTRODUÇÃO}

As alterações estéticas no abdome estão entre as queixas mais frequentes das mulheres. Destacam-se o acúmulo de gordura localizada, que pode estar presente desde a adolescência, aumentando com a idade, e alterações pósgestacionais, com excesso de pele e diástase de músculos reto-abdominais. Fatores genéticos, flutuações de peso e sedentarismo também alteram o aspecto estético do abdome ao longo da vida.

A cirurgia plástica do abdome é feita há mais de um século, compreendendo plicatura dos músculos reto-abdominais, transposição do umbigo para o retalho cutâneo descolado e terminando com cicatriz final na pele em sulco abdominal inferior $^{1-3}$. A lipoaspiração, introduzida posteriormente, permitiu que se melhorasse o contorno abdominal sem a realização da ressecção de pele, e é especialmente útil em pacientes nuligestas ${ }^{5}$. Nesta última década, ganhou espaço a lipoabdominoplastia, cirurgia que associa ambos os procedimentos em um só tempo cirúrgico ${ }^{4}$.

Apesar dos avanços técnicos e da satisfação geralmente referida pelas pacientes, a avaliação dos resultados após uma cirurgia estética é ainda difícil, de caráter pouco científico, e o que temos são critérios, na maioria das vezes, subjetivos ${ }^{5,6}$. Ferreira propôs, em 2000, uma escala para avaliação do resultado estético após cirurgia de redução mamária baseada em cinco critérios visuais (volume, forma, simetria, aréola e cicatriz), tornando mais objetiva a opinião do avaliador, e facilitando a realização de estudos comparativos e análises estatísticas ${ }^{7}$. Essa escala mostrou-se útil também na avaliação de resultados de cirurgias de mastoplastia de aumento, mesmo após perda ponderal. Destacam-se a simplicidade para sua execução, e a possibilidade de ser realizada com a opinião da própria paciente no momento da consulta, ou posteriormente por avaliação fotográfica ${ }^{8}$.

O objetivo do presente estudo é apresentar uma escala semelhante para a avaliação padronizada de resultados estéticos de cirurgias plásticas no abdome.

\section{MÉTODO}

A avaliação pode ser feita diretamente com a paciente, ou por meio de fotografias. Neste caso, organizam-se em um diapositivo as fotografias da paciente nas posições frente, perfil e duas oblíquas, no pré-operatório, e em outro diapositivo, as fotografias nas mesmas posições, no pós-operatório. Os avaliadores preferivelmente deverão ser independentes, mas pode ser a própria paciente ou o próprio cirurgião.

São atribuídas notas para cada um dos seguintes parâmetros visuais: volume do abdome, contorno lateral, flacidez/ excesso de pele, aspecto do umbigo e qualidade da cicatriz em parede abdominal, segundo a escala de avaliação, simplificadamente: $0=$ insatisfatório, $1=$ regular, $2=$ bom e/ou cicatriz inexistente.

É fornecida ao avaliador uma tabela explicativa orientando a pontuação sobre cada parâmetro (Quadro 1). A soma da nota dos parâmetros confere a nota final, que pode variar de 0 a 10.

Com finalidade de avaliação para trabalhos científicos, idealmente, três avaliadores cirurgiões plásticos não participantes do procedimento cirúrgico devem fazer uma avaliação individual utilizando a escala, sem identificação das pacientes ou do momento de avaliação (pré ou pós-operatório) - Figura 1. As médias dos resultados estéticos podem ser comparadas entre dois grupos que realizaram, por exemplo, técnicas diferentes.

\section{DISCUSSÃO}

As expectativas das pacientes em relação ao resultado pós-operatório de uma cirurgia estética costumam ser bastante elevadas, e cabe ao cirurgião fazer com que estas estejam cientes das limitações cirúrgicas decorrentes de suas características anatômicas no pré-operatório ${ }^{5,7}$. Parâmetros objetivos simples e de fácil compreensão e reprodução, como os utilizados na escala apresentada no presente estudo, podem auxiliar o cirurgião nesta tarefa. Os cinco itens utilizados representam o pensamento intuitivo de todo cirurgião ao fazer a avaliação de uma paciente que se apresenta com queixa estética na região do abdome: avalia-se visualmente a relação conteúdo-continente (quantidade de panículo adiposo vs. presença de excesso de pele), o contorno lateral, o aspecto da cicatriz umbilical e a presença de cicatrizes no abdome. A realização desta avaliação pode ser feita diretamente com a paciente, tanto no pré quanto no pós-operatório, documentando de forma objetiva a melhora proporcionada pelo procedimento cirúrgico. Este tipo de avaliação de resultados estéticos e sua documentação em prontuário podem inclusive ser úteis em processos médico-legais. É comum certas pacientes ganharem peso após uma cirurgia estética, sugerindo a utilidade do registro em prontuário de uma avaliação precoce, por exemplo, cerca de três meses após o procedimento.

A maioria dos estudos na literatura apresenta a avaliação de resultados após cirurgia estética de forma subjetiva, tais como escalas de mudança cosmética de quatro gradações, ou uma única escala global de "zero a dez"6. A confiabilidade destes métodos é inaceitável, segundo Kim et al. ${ }^{9}$, para quem escalas globais sofrem devido à terminologia vaga, apontando a necessidade de medidas separadas para diferentes fatores (itens) da avaliação estética, a fim de que os resultados sejam mais fidedignos.

Foi também demonstrado anteriormente que a avaliação de resultados por meio da análise de fotografias apresenta resultados comparáveis aos da avaliação realizada durante 
Quadro 1 - Quadro explicativo orientando a pontuação sobre cada parâmetro de avaliação do abdome, com variação, simplificadamente, "0 - insatisfatório", "1 - regular" e "2 - bom". A soma da nota dos parâmetros confere a nota final, que pode variar de 0 a 10.

\begin{tabular}{|c|c|}
\hline Nome & Descrição \\
\hline & Volume do abdome \\
\hline 0 & Grande quantidade de gordura no abdome, grande abaulamento \\
\hline 1 & Moderada quantidade no abdome, abaulamento regular \\
\hline \multirow[t]{2}{*}{2} & Quantidade de tecido gorduroso adequada à estrutura da paciente, sem abaulamento \\
\hline & Contorno lateral \\
\hline 0 & Em visão anterior, cintura abdominal ao nível do umbigo com abaulamento \\
\hline 1 & Abdome com contorno lateral reto, sem definição da cintura \\
\hline \multirow[t]{2}{*}{2} & Cintura abdominal bem definida, com concavidade \\
\hline & Excesso de pele/flacidez \\
\hline 0 & Grande excedente de pele, com flacidez e estrias \\
\hline 1 & Quantidade moderada de pele excedente, com ou sem estrias e flacidez \\
\hline \multirow[t]{2}{*}{2} & Sem excesso de pele e/ou flacidez \\
\hline & Aspecto do umbigo \\
\hline 0 & Cicatriz umbilical com desvios, retrações, hipertrofias ou excesso de pele adjacente \\
\hline 1 & Aspecto aceitável, com ou sem discretos desvios, retrações, excesso de pele ou cicatriz \\
\hline \multirow[t]{2}{*}{2} & Cicatriz umbilical de aspecto natural \\
\hline & Cicatrizes em parede abdominal \\
\hline 1 & Cicatriz hipertrófica ou queloideana, hipercrômica, hipocrômica, deprimida ou em posição inadequada \\
\hline 1 & Cicatriz em abdome de aspecto regular \\
\hline 2 & Ausência de cicatriz em abdome, ou cicatriz de bom aspecto, quase imperceptível \\
\hline
\end{tabular}
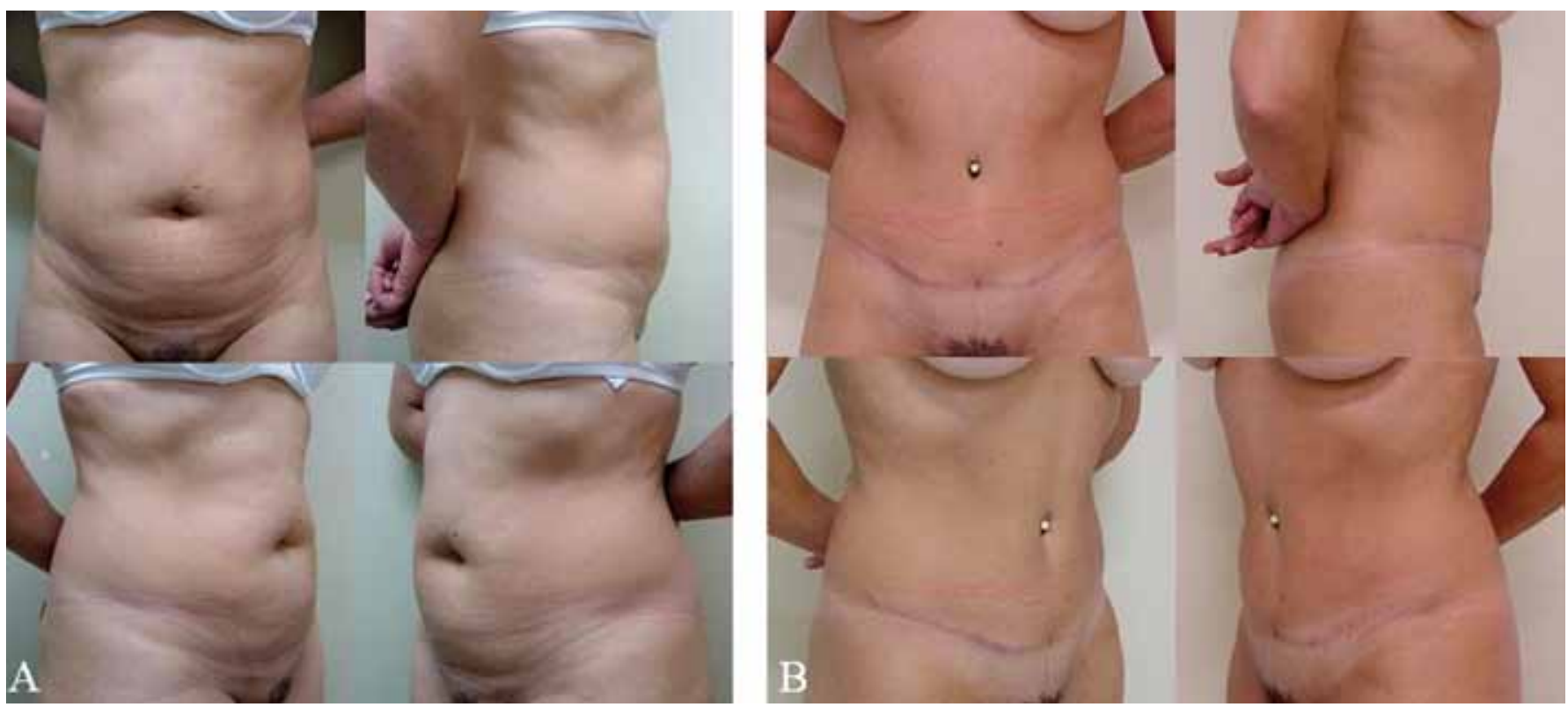

Figura 1 - Paciente de 34 anos submetida à abdominoplastia tradicional. A: Pré-operatório, nota média 3,67, de acordo com os avaliadores independentes. B: Pós-operatório, nota média 8,00. 
o exame físico, sendo aceitáveis tanto imagens impressas quanto apresentadas em um monitor de computador, sendo inclusive essa forma menos invasiva para a paciente ${ }^{9,10}$.

A soma da avaliação dos cinco aspectos da presente escala, que pode variar de zero a 10 pontos, expressa de forma mais padronizada e objetiva o grau de satisfação do avaliador com a aparência estética do abdome, seja este avaliador a própria paciente ou o profissional. Uma nota final entre 0 e 4 no pós-operatório indicaria um resultado pobre ${ }^{7}$. Porém, mais importante que a nota final, é o ganho estético em relação à nota inicial que deve ser analisado.

A escala apresentada pode ser utilizada em estudos para comparar diferentes técnicas cirúrgicas, ou mesmo para padronizar resultados apresentados em Congressos ou publicações científicas.

\section{REFERÊNCIAS}

1. Callia WEP. Contribuição para o estudo da correção cirúrgica do abdome em pêndulo e globoso: técnica original [Tese de Doutorado]. São Paulo:Faculdade de Medicina, Universidade de São Paulo;1965.
2. Pitanguy I. Abdominal lipectomy: an approach to it through an analysis of 300 consecutive cases. Plast Reconstr Surg. 1967;40(4):384-91.

3. Illouz YG. Une nouvelle technique pour les lipodystrophies localisées. Rev Chir Esth Franc. 1980;6(9):Ap.

4. Saldanha OR, De Souza Pinto EB, Mattos WN Jr, Pazetti CE, Lopes Bello EM, Rojas Y, et al. Lipoabdominoplasty with selective and safe undermining. Aesthetic Plast Surg. 2003;27(4):322-7.

5. Young VL, Nemecek JR, Nemecek DA. The efficacy of breast augmentation: breast size increase, patient satisfaction, and psychological effects. Plast Reconstr Surg. 1994;94(7):958-69.

6. Strasser EJ. An objective grading system for the evaluation of cosmetic surgical results. Plast Reconstr Surg. 1999;104(7):2282-5.

7. Ferreira MC. Evaluation of results in aesthetic plastic surgery: preliminary observations on mammaplasty. Plast Reconstr Surg. 2000;106(7):1630-5.

8. Cintra Junior W. Mastopexia com inclusão de implantes mamários após tratamento cirúrgico da obesidade mórbida: avaliação da satisfação das pacientes e resultados cirúrgicos [Tese de Doutorado]. São Paulo: Universidade de São Paulo, Faculdade de Medicina;2009. 123p.

9. Kim MS, Sbalchiero JC, Reece GP, Miller MJ, Beahm EK, Markey MK. Assessment of breast aesthetics. Plast Reconstr Surg. 2008;121(4):186e94e.

10. Christie DR, O'Brien MY, Christie JA, Kron T, Ferguson SA, Hamilton $\mathrm{CS}$, et al. A comparison of methods of cosmetic assessment in breast conservation treatment. Breast. 1996;5(5):358-67.

Correspondência para:

Alessandra Grassi Salles

Rua Joaquim Floriano, 466, cj. 2102 - São Paulo, SP, Brasil - CEP 04534-002

E-mail: agsalles@uol.com.br 